\title{
Pravastatin Improves Angiogenic Factors and Feto- Placental Doppler in Pregnancy with Early Severe Fetal Growth Restriction: A Case Report
}

\author{
Erika Bonacina $^{1}$, Raquel Ferrer-Oliveras ${ }^{1}$, Manel Mendoza ${ }^{1 *}$, Elena Carreras ${ }^{1 * *}$ and Jaume Alijotas-Reig ${ }^{2 * *}$ \\ ${ }^{1}$ Maternal Fetal Medicine Unit, Department of Obstetrics, Hospital Universitari Vall d'Hebron, Universitat Autonoma de Barcelona, Spain \\ ${ }^{2}$ Systemic Autoimmune Diseases Unit, Department of Medicine, Hospital Universitari Vall d'Hebron, Universitat Autonoma de Barcelona, Spain
}

${ }^{* *} E C$ and JAR contributed equally

*Corresponding author: Manel Mendoza, Department of Obstetrics, Maternal Fetal Medicine Unit, Hospital Universitari Vall d'Hebron. Universitat Autonoma de Barcelona, Passeig de la Vall d'Hebron, 119-129, 08035 Barcelona, Spain.

Received Date: December 09, 2020

Published Date: January 05, 2021

\begin{abstract}
Early fetal growth restriction (FGR) is a leading cause of perinatal morbidity and mortality. No therapeutic strategies have proved to be effective in improving fetal and neonatal outcomes either in growth restricted fetuses or any placenta-related disorder such as preeclampsia. Delivery of the fetus and placenta is the only definitive cure. However, recent studies suggest that pravastatin appears to be safe in pregnancy, improves placental perfusion and fetal growth and prevents angiogenic imbalance. Angiogenic (PIGF) and antiangiogenic (sFlt-1) factors and feto-maternal Doppler evaluation are strongly associated with fetal growth restriction prognosis and severity. Thus, pravastatin has been proposed as a promising therapeutic drug for fetal growth restriction. We report the findings of a case of early-onset FGR treated with pravastatin 40mg daily until delivery. During this time, feto-maternal Doppler and angiogenic profile evolution were recorded. Fetal and placental perfusion observed on Doppler and angiogenic imbalance improved after pravastatin was started. sFlt-1 down-regulation was more marked than PlGF up-regulation. The patient developed severe preeclampsia (PE) at 27+6 weeks of gestation, necessitating immediate fetal extraction owing to hypertension refractory to adequate treatment. This report supports further investigation on the use of pravastatin to improve angiogenic profile and feto-maternal circulation in FGR fetuses.
\end{abstract}

Keywords: Fetal growth restriction; Pravastatin; PIGF, sFlt-1; Feto-placental Doppler

\section{Introduction}

Fetal growth restriction (FGR) affects $7-10 \%$ of all pregnancies and refers to a fetus that has failed to achieve its biological growth potential [1]. Growth-restricted fetuses have a 5- to 10-fold risk of dying in utero and a higher risk of perinatal morbidity and mortality [2]. Abnormal placentation is a key factor in fetal growth restriction pathophysiology [3]. Several histopathologic features such as villous infarction, maternal vascular changes and villous morphologic alterations are observed more frequently in placentas from pregnancies complicated by FGR [4]. Placental function evaluation by umbilical artery (UA) Doppler velocimetry is the clinical standard for identifying early-onset FGR [1-3] and there is evidence that its use in these pregnancies improves a number of obstetric care outcomes and reduces perinatal deaths [4].

Fetal deterioration latency can vary from case to case; however, it usually lasts weeks and often follows a series of changes reflected in a sequential pattern on Doppler that permits tailoring of fetal monitoring and elective delivery [1].

Uteroplacental insufficiency is associated with a predominantly antiangiogenic environment characterized by up-regulation of soluble fms-like tyrosine kinase-1 (sFlt-1) and down-regulation of placental growth factor (PlGF) [5]. Abnormal angiogenic factor 
levels in the maternal circulation play a key pathogenic role in the development of endothelial dysfunction with ensuing preeclampsia [5]. The value of angiogenic biomarkers in the prediction, prognosis and characterization of early-onset preeclampsia and FGR has been demonstrated in several studies [5,6]. In uncomplicated pregnancies, PlGF rises until the third trimester of pregnancy and falls thereafter whereas sFlt-1 levels rise as the pregnancy progresses. Thus, the sFlt-1 to PIGF ratio rises with gestational age. These changes are more patent in pregnancies that will eventually develop placental disease [5-7].

No treatment currently exists for placental insufficiency and delivery is the only way to stop disease progression. If preeclampsia or FGR occur early in gestation, the need to deliver the fetus preterm for maternal and/or fetal indications can lead to neonatal death and disability arising from prematurity.

Some studies suggest that statins could be a promising therapeutic or preventive strategy for placental insufficiency $[8,9]$. Significant clinical benefits and improvement in the angiogenic profile have been reported in patients with early-onset preeclampsia [10] and umbilical artery flow in twin pregnancies with discordant fetal growth seems to improve after pravastatin is started [11]; however, no previous studies reported the effect of pravastatin on the uterine artery Doppler and angiogenic factors of FGR single pregnancies in humans.

\section{Case Report}

\section{Case description}

A 41-year-old woman with early-onset FGR was referred to our center at $25+4$ weeks of gestation (WG). Estimated fetal weight (EFW) at admission $(25+4$ WG) was 429 grams (g) that corresponded to a zero percentile. After the diagnosis of FGR was confirmed, the patient received a course of antenatal corticosteroids for fetal maturation, amniocentesis was performed and prophylactic low-molecular-weight heparin was initiated owing to the presence of 3 other thrombotic risk factors (obesity, smoking and age $>40$ years). Negative results were obtained in tests for antiphospholipid antibodies. These tests were repeated on two occasions during pregnancy. Results of the amniocentesis and Doppler findings were suggestive of FGR secondary to severe placental insufficiency (increased pulsatility of the uterine arteries with present bilateral early diastolic notches, normal fetal CGH arrays and negative polymerase chain reaction for cytomegalovirus in amniotic fluid analysis).

The patient was offered treatment with pravastatin and, after being informed of its risks and benefits, consented to its use. Pravastatin was started at $26+2$ WG.

This patient was the first to be enrolled in a pilot study where patients presenting FGR before 28+0 WG were offered pravastatin $40 \mathrm{mg}$ daily until delivery. The aim of that study was to ascertain whether pravastatin treatment exerted beneficial effects on growthrestricted fetuses. FGR was defined as estimated fetal weight below the third centile or below the 10th centile associated with any fetoplacental Doppler impairment.

The effects of pravastatin were evaluated on feto-maternal Doppler ultrasound (umbilical artery, middle cerebral artery, ductus venosus and uterine arteries) together with serum angiogenic factors (sFlt-1 and PlGF), clinical and biochemical findings related to preeclampsia such as mean systolic and diastolic blood pressures, qualitative proteinuria, 24-hour quantitative proteinuria, prodromal symptoms of eclampsia (headache, visual disturbance, tinnitus, epigastric pain and/or upper right abdominal pain) and other relevant laboratory findings.

\section{Findings}

The findings are summarized in Figure 1. No significant effects were observed on any biochemical parameters, blood pressure or middle cerebral artery (MCA) Doppler after pravastatin was started. However, an effect on umbilical artery (UA) pulsatility index (PI), uterine artery (UtA) PI and angiogenic factors was observed after 36 hours. Mean UtA PI dropped significantly (from 3.34 to 2.64, although being both above the 99th percentile for gestational age) and this improvement persisted 7 days later. UA PI had been rising progressively prior to pravastatin and remained around normal values thereafter.

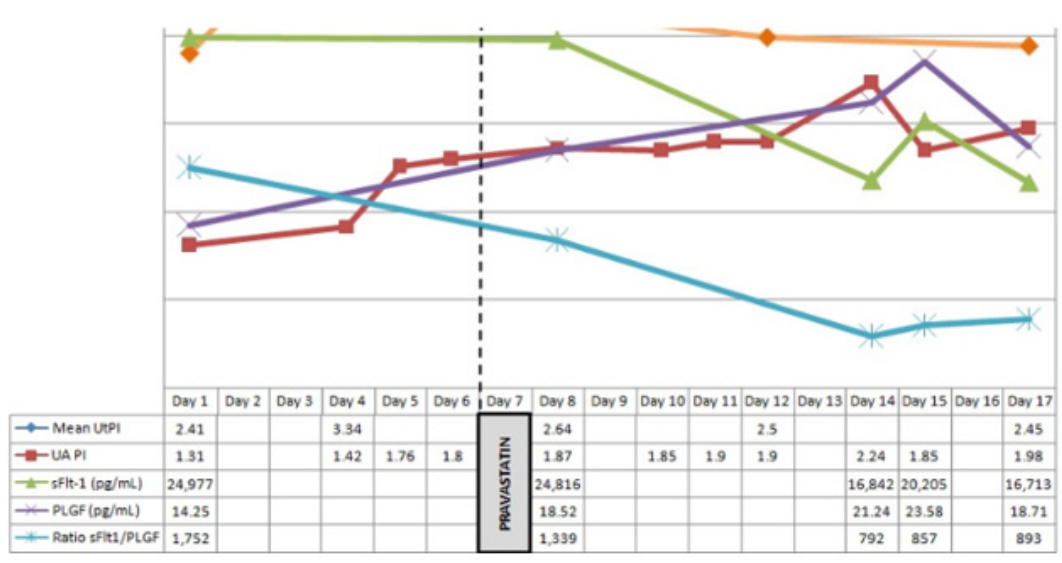

Figure 1: Evolution of feto-placental Doppler and angiogenic factors. 
Regarding angiogenic factors, an improvement was observed in the angiogenic profile consisting of an increase in the angiogenic factor (PLGF), a reduction in the antiangiogenic factor (sFlt-1) and thus a lower and more favorable sFlt-1 to PLGF ratio. The effect was more marked on sFlt-1 than PLGF. The sFlt-1 to PLGF ratio prior to treatment was 1,752 and 1,339 after 36 hours of treatment. The lowest sFlt- 1 to PLGF ratio values were observed 6 days after pravastatin was started and remained steady, around 800, until delivery 5 days later (Figure 1).

\section{Outcome}

The patient developed mild preeclampsia (new-onset hypertension and proteinuria $>300 \mathrm{mg}$ in 24 hours) at $27+2 \mathrm{WG}$ Four days later, she presented features of severe preeclampsia (PE) consisting of persistent headache and hypertension > 160/110 mmHg. The diagnosis of severe PE was established 11 days after treatment and 17 days after the diagnosis of FGR and the first sFlt-1 to PLGF ratio measurement $(1,752)$. Cesarean section was performed at $27+6 \mathrm{WG}$ owing to persistent hypertension refractory to adequate pharmacologic treatment. A 512g female newborn was delivered with an Apgar score of 7/8/9 and umbilical artery $\mathrm{pH}$ of 7.25. The infant was admitted to the neonatal intensive care unit (NICU) for 144 days; she presented severe complications such as sepsis and required respiratory support for 88 days. After discharge, she evolved well with no further complications.

\section{Discussion}

FGR has two different phenotypes: early- or late-onset in gestation. In general, but not always, early onset correlates with the most severe forms of FGR. Early-onset FGR accounts for $20-30 \%$ of all FGR and is associated with early-onset PE in up to $50 \%$ of cases [1]. Early-onset FGR is closely associated with severe placental insufficiency and chronic fetal hypoxia $[2,4]$.

Placental function evaluation by umbilical artery (UA) Doppler velocimetry is the standard technique for identifying early-onset FGR and evidence exists of its use in improving obstetric outcomes and reducing the perinatal death rate in these pregnancies [12].

In a normal pregnancy, flow impedance in the uterine arteries diminishes with gestation, and histopathologic studies suggest that this is due to trophoblastic invasion of the spiral arteries and their conversion into low-resistance vessels [13]. Trophoblastic invasion failure is associated with uteroplacental insufficiency complications. Doppler ultrasound of the uterine arteries provides a proxy measure of physiologic placental remodeling. When trophoblastic invasion has not occurred, end-diastolic flow remains unaltered and a diastolic notch is often present [14]. Over the last decade, research has shown that uterine artery Doppler early in pregnancy is able to identify patients at risk of PE and FGR [14,15]. Increased resistance of UtA as the pregnancy progresses has been associated with a greater risk of PE [15].

Angiogenic status in pregnancy is closely associated with time from diagnosis to delivery. Some sFlt-1 to PLGF ratio cut-offs have been established to predict the need for delivery in patients with suspected PE [16]. Below $34 \mathrm{WG}$, patients with an sFlt-1 to PlGF ratio $>655.2$ are at higher risk of imminent delivery. In the study by Stepan H, et al. approximately $30 \%$ of patients remained pregnant after 48 hours and only $6 \%$ were still pregnant after 7 days [16].

No treatment exists for placental insufficiency and delivery is the only way to halt disease progression [1-3]. When PE or FGR occur early in pregnancy, they can lead to neonatal morbidity and mortality. Thus, an efficacious therapy to slow disease progression would improve maternal and perinatal outcomes.

Consequently, interest has arisen recently in the use of statins to treat preeclampsia. Unfortunately, statin use in pregnancy has an FDA category $\mathrm{X}$ rating owing to results of observational studies demonstrating an association with fetal malformations if administered during the first trimester [17]. However, pravastatin may be a promising therapeutic candidate given that its safety profile is better than that of other statins.

Unlike other statins, which are hydrophobic, pravastatin is hydrophilic, meaning that it may less readily pass through the placenta to the fetus [18]. Cohort studies have shown that hydrophilic statins, including pravastatin, are not associated with an increased risk of fetal malformations [10]. Moreover, inhibition of HMG-CoA reductase by pravastatin is not as great as that of other statins; pravastatin is cleared through both renal and hepatic routes and is not dependent on CYP3A metabolism [9]. Additionally, recent transplacental studies showed the ability of pravastatin to cross the placenta to be limited and clearance higher from fetus to mother than mother to fetus [18].

In vitro, statins have been shown to reduce sFlt-1 secretion from endothelial cells, trophoblasts and preterm preeclamptic placental explants [19]. In mice with an sFlt-1-induced preeclampsia-like syndrome, pravastatin treatment lowered sFlt-1 concentrations to levels similar to those of controls. Placental PLGF expression was upregulated and hypoxia markers downregulated to levels similar to controls [20].

Statins have been proposed for treating and/or preventing obstetric complications in patients at high risk of placental disease; however, only one study, by Lefkou, et al. in a small observational trial, evaluated pravastatin treatment in growth-restricted fetuses and reported a dramatic improvement in both maternal and fetal/ neonatal outcomes in women with antiphospholipid syndrome (APS) given pravastatin after the onset of preeclampsia and/or IUGR compared with controls. No information on the effects of the drug on the angiogenic profile was provided in that study [21] and no other studies have evaluated the pravastatin effect on angiogenic factors in FGR; however, a multicenter randomized controlled trial showed an improvement in the angiogenic profile, by lowering sFlt1 levels, in women with preterm PE [17]. 


\section{Conclusions}

To our knowledge, no previous studies evaluated the effect of pravastatin on maternal serum sFlt1 and PlGF levels and Doppler values in patients with fetal growth restriction with or without APS.

Early evidence of the impact of pravastatin on angiogenic imbalance and feto-placental Doppler in pregnant women carrying an FGR fetus is reported. This study supports a likely benefit of pravastatin for FGR fetuses without APS by improving the angiogenic profile and feto-maternal circulation, which correlates with disease severity and progression velocity. The case reported in this article shows that the duration of the pregnancy is almost two times longer than expected based on the study published by Stepan H, et al [16]. Thus, it is plausible to believe that pravastatin could extend pregnancy and reduce extreme prematurity-related perinatal morbidity and mortality; however, this hypothesis cannot be ascertained in this study as there are no control patients to compare outcomes.

\section{Acknowledgment}

Assistance provided by the Clinical Pharmacology service at Hospital Universitari Vall d'Hebron was greatly appreciated.

\section{Conflict of Interest}

Authors declare no conflict of interest.

\section{References}

1. Figueras F, Gratacós E (2014) Update on the diagnosis and classification of fetal growth restriction and proposal of a stage-based management protocol. Fetal Diagn Ther 36(2): 86-98.

2. McIntire DD, Bloom SL, Casey BM, Leveno KJ (1999) Birth weight in relation to morbidity and mortality among newborn infants. N Engl J Med 340(16): 1234-1238.

3. Altshuler G, Russell P, Ermocilla R (1975) The placental pathology of small-for-gestational age infants. Am J Obstet Gynecol 121(3): 351-359.

4. Mifsud W, Sebire NJ (2014) Placental pathology in early-onset and lateonset fetal growth restriction. Fetal Diagn Ther 36(2): 117-128.

5. Levine RJ, Maynard SE, Qian C, Lim K-H, England LJ, et al. (2004) Circulating angiogenic factors and the risk of preeclampsia. The New England Journal of Medicine 350: 672-683.

6. Crispi F, Llurba E, Domínguez C, Martín-Gallán P, Cabero L, et al. (2008) Predictive value of angiogenic factors and uterine artery Doppler for early- versus late-onset pre-eclampsia and intrauterine growth restriction. Ultrasound Obstet Gynecol 31(3): 303-309.

7. Romero R, Nien JK, Espinoza J, Todem D, Fu W, et al. (2008) A longitudinal study of angiogenic (placental growth factor) and antiangiogenic (soluble endoglin and soluble vascular endothelial growth factor receptor-1) factors in normal pregnancy and patients destined to develop preeclampsia and deliver a small for gestational age neonate. J Matern Fetal Neonatal Med 21(1): 9-23.

8. Lecarpentier E, Morel O, Fournier T, Elefant E, Chavatte-Palmer P, et al. (2012) Statins and pregnancy: between supposed risks and theoretical benefits. Drugs 72(6): 773-788.

9. Costantine MM (2016) Pravastatin to prevent obstetrical complications in women with antiphospholipid syndrome. J Clin Invest 126(8): 2792 2794.

10. Brownfoot FC, Tong S, Hannan NJ, Binder NK, Walker SP, et al. (2015) Effects of Pravastatin on Human Placenta, Endothelium, and Women With Severe Preeclampsia. Hypertension 66(3): 687-697.

11. Jurisic A, Jurisic Z, Lefkou E, Pombo J, Girardi G (2018) Pravastatin and-L-arginine combination improves umbilical artery blood flow and neonatal outcomes in dichorionic twin pregnancies through an nitric oxide-dependent vasorelaxant effect. Vascul Pharmacol 110: 64-70.

12. Neilson JP, Alfirevic Z (2000) Doppler ultrasound for fetal assessment in high risk pregnancies. Cochrane Database Syst Rev CD000073.

13. Lin S, Shimizu I, Suehara N, Nakayama M, Aono T (1995) Uterine artery Doppler velocimetry in relation to trophoblast migration into the myometrium of the placental bed. Obstet Gynecol 85 (5 Pt 1): 760-765.

14. Papageorghiou AT, Yu CKH, Nicolaides KH (2004) The role of uterine artery Doppler in predicting adverse pregnancy outcome. Best Pract Res Clin Obstet Gynaecol 18(3): 383-396.

15. Llurba E, Turan O, Kasdaglis T, Harman CR, Baschat AA (2013) Emergence of late-onset placental dysfunction: relationship to the change in uterine artery blood flow resistance between the first and third trimesters. Am J Perinatol 30(6): 505-512.

16. Stepan H, Herraiz I, Schlembach D, Verlohren S, Brennecke S, et al. (2015) Implementation of the sFlt-1/PlGF ratio for prediction and diagnosis of pre-eclampsia in singleton pregnancy: implications for clinical practice. Ultrasound Obstet Gynecol 45(3): 241-416.

17. Costantine MM, Cleary K, Hebert MF, Ahmed MS, Brown LM, et al. (2016) Safety and pharmacokinetics of pravastatin used for the prevention of preeclampsia in high-risk pregnant women: a pilot randomized controlled trial. Am J Obstet Gynecol 214(6): 720.

18. Nanovskaya TN, Patrikeeva SL, Paul J, Costantine MM, Hankins GDV, et al. (2013) Transplacental transfer and distribution of pravastatin. Am J Obstet Gynecol 209(4): 373.

19. Brownfoot FC, Tong S, Hannan NJ, Hastie R, Cannon P, et al. (2016) Effects of simvastatin, rosuvastatin and pravastatin on soluble fms-like tyrosine kinase 1 (sFlt-1) and soluble endoglin (sENG) secretion from human umbilical vein endothelial cells, primary trophoblast cells and placenta. BMC Pregnancy Childbirth 16: 117.

20. Saad AF, Kechichian T, Yin H, Sbrana E, Longo M, et al. (2014) Effects of pravastatin on angiogenic and placental hypoxic imbalance in a mouse model of preeclampsia. Reprod Sci 21(1): 138-145.

21. Lefkou E, Mamopoulos A, Dagklis T, Vosnakis C, Rousso D, et al. (2016) Pravastatin improves pregnancy outcomes in obstetric antiphospholipid syndrome refractory to antithrombotic therapy. J Clin Invest 126(8): 2933-1240. 